Article

\title{
The regulation effect of crude polysaccharides from Cordyceps militaris on obesity control and gut microbiota community via high-fat diet-fed mice model
}

\author{
Minglei Yu 1,2, Nan Hui 1,2, Kashif Hayat 1,2, Xijia Yang 1,2, Shaohua Chu 1,2, Dan Zhang 1,2, Juncai \\ Wang ${ }^{1,2}$, Xunfeng Chen ${ }^{1,2}$, Jina Ding ${ }^{1,2}$, Yaowei Chi ${ }^{1,2}$ and Pei Zhou 1,2,3,* \\ 1 School of Agriculture and Biology, Shanghai Jiao Tong University, Shanghai 200240, China \\ 2 Key Laboratory of Urban Agriculture, Ministry of Agriculture, Shanghai, 200240, China; \\ fish_sunshine@sjtu.edu.cn (M.Y.); nan.hui@sjtu.edu.cn (N.H.); khayat97@sjtu.edu.cn (K.H.); \\ eileenyang33@163.com (X.Y.); chushyt@sjtu.edu.cn (S.C.); zhdsjtu@sjtu.edu.cn (D.Z.); \\ wangjuncai@sjtu.edu.cn (J.W.); chenxunfeng@sjtu.edu.cn (X.C.); dingjina1217@126.com (J.D.); \\ cyw8103@163.com (Y.C.) \\ 3 Bor S. Luh Food Safety Research Center, Shanghai Jiao Tong University, Shanghai, 200240, China \\ * Correspondence: zhoupei@sjtu.edu.cn
}

\begin{abstract}
Administration of crude polysaccharides extract from natural product is a promising gut microbiota-targeted approach to preventing obesity and associated metabolic disorders. Dietary restrictions can change the type and number of gut bacteria, which is an important factor in delaying the onset and burden of diseases. This study aimed to investigate the effects of high-yield crude polysaccharides from Cordyceps militaris (CMP) on high-fat diet (HFD) mice model and the gut microbiota community assembly, and to identify whether selenium (Se) addition would improve $\mathrm{CMP}$ action mode during cultivation. We found that the CMP treatment ameliorated adipose and liver pathologic morphology and fat accumulation in obese mice, while, SeCMP intervention was not superior than CMP in body mass gain, but notably decreasing serum triglyceride level increased by HFD. The upregulated expression of gene Cyp7a1 in liver and protein UCP1 in brown adipose tissue (BAT) preliminary indicated that the effect might relate to bile acids (BAs) metabolism pathway and thermogenesis. In addition, CMP showed a drastic decrease in the gut microbes which positively correlated with dyslipidemia parameters. Our result reveals the potential of CMP to be used as functional food in the prevention of diet-induced adipose and liver steatosis, so does SeCMP has outstanding capacity of improving dyslipidemia.
\end{abstract}

Keywords: Cordyceps militaris; crude polysaccharides; selenium-rich crude polysaccharides; highfat diet (HFD); obesity; dyslipidemia; hypertriglyceridemia; gut microbiota

\section{Introduction}

Small molecule organic compounds predominate in the list of U.S. Food and Drug Administration (FDA) approved as obesity prevention medications. However, these managements have large variations in efficacy in different individuals and severe side effect, along with environmental issues, due to a large number of organic solvents used in the preparation process [13]. It is very challenging to develop effective, safe and inexpensive therapies as alternative to present drugs. Accumulating evidence has proved water-soluble substances derived from plants and mushrooms exhibited health benefits relating to the incidence of obesity, type- 2 diabetes, cardiovascular disease and cancer. These effects are due to the high content of fiber, phytochemicals, vitamins and minerals [4]. Polysaccharide is a kind of indigestible fiber and is supposed to develop into anti-obesogenic supplements because they can ameliorate pathological characteristics and restore gut microbiota community [5-8]. However, isolates from extensive sources differ in internal 
mode of action, and questions remain about the effectiveness, mode of action and safety of these potential supplements and functional foods.

Cordyceps sinensis is used as a tonic in Asian countries. Water extract and a $300 \mathrm{kDa}-$ polysaccharide $\mathrm{H} 1$ from $C$. sinensis have had observed anti-obesogenic effect at a surprising low dosage of $20 \mathrm{mg} / \mathrm{kg}$ bw in HFD-fed mice [9]. Plant and fungi water extract fraction usually take effect in vivo at $100-1000 \mathrm{mg} / \mathrm{kg}$ bw/day dosage $[5,10,11]$. Although C. sinensis extract is a promising for developing obesity prevention functional foods to some extent, overexploitation and climate change has generated extensive concern over the sustainability of the collection [12]. Extensive research has focused on a cultured Cordyceps species -- Cordyceps militaris, which is composed with various bioactive compounds, including cordycepin, adenosine, guanosine, hypoxanthine, uridine, adenine [13]. C. militaris and its extracts have potential health effects, such as cell proliferation [14, 15], diabetic nephropathy [16], immunomodulatory, anti-oxidative activity [17], etc. Many reports also evidenced that small molecules in C. militaris can reduce obesity. Cordycepin is capable of reducing fat storage and lipid metabolism [18, 19]. Kim et al. [20] reported administration of 50\% ethanol extract from $C$. militaris reduced body weight, fat accumulation and hyperlipidemia in HFD-induced obese mice. One question arising from these observations is, what about the effect of water-soluble profile of $C$. militaris on obese mice model? Hence, it is of great interest to explore the in vivo anti-obesogenic effect of $C$. militaris crude polysaccharides.

Accumulating evidence has proved the mechanism of anti-obesogenic effect is partially attributed to shift the community and function of the gut microbiota [21]. Gut microorganisms use ingested fiber and polysaccharides for fundamental biological processes, and the metabolic outputs of those processes serve as signaling molecules in the lipid metabolism of the host. The dietmicrobiota interaction control appetite, nutrient digestion and absorption, lipid metabolic pathways, energy expenditure and inflammatory generation [4]. Parabacteroides goldsteinii was a probiotic generated by supplementing $C$. sinensis improving insulin sensitivity and anti-inflammation [9]. Supplementation with Ganodorma lucidum extract prevents endotoxin producing bacteria release proinflammatory cytokines (such as lipopolysaccharide, LPS) translocating from intestine to the blood [8]. Nowadays, the advent of high-throughput sequencing combined with bioinformatics substantially boosted our ability to study complex microbial communities and identify bacterial biomarkers that can predict individual's response to treatment. Despite the intestinal microbial communities has been screened in mice supplied with $C$. sinensis polysaccharides $[9,22]$, the effect of cultured C. militaris crude polysaccharides (CMP) remains unclear. Cultured C. militaris is a promising alternative for large-scale isolation of effective bioactive ingredients at low-cost [23-25], and the solvent includes only water and ethanol used in the extraction process is environmentalfriendly, revealing the gut microbiota may shed some lights on uncovering CMP anti-obesogenic mechanism at a new perspective.

Selenium (Se) is an essential trace element for the development and maintenance human health associating with its antioxidant property [26]. Recently, Se supplements are a potential prebiotic to treat obesity. Although some researchers have uncovered the anti-obesity effect of Se protein, Seenriched bacterial, and Se-rich commercial product, however, few has demonstrated the action of Serich crude polysaccharide in the treatment of obesity. Bifidobacterium longum DD98 fermented with Se in culture medium obtained Se- $B$. longum DD98 exhibited stronger anti-obesity effect than Na2SeO3, SeMet, B. longum DD98 and B. longum DD98 + SeMet groups [27]. Surhio et al. [28] found Se modified exopolysaccharide of Lachnum sp. SeLEP-1b show better effect on body weight reduction, serum and liver lipids regulation, atherogenic index, and enhanced the activities of antioxidant enzymes of hyperlipidemic mice than LEP-1b. These results suggested Se combined biological substances complex have stronger pharmacological activities than normal type, but this is not always the case. SD rats fed with Se-rich black tea powder did not exhibit better effects in preventing body weight gain, fat accumulation and attenuating gut microbiota dysbiosis than Se-deficient one, although it showed stronger activity in decreasing fasting serum glucose and increasing insulin sensitivity [29]. Thus, it is worthy to evaluate therapeutic value of Se-rich crude polysaccharides in obese murine models. 
To assess the effect of high-yield crude polysaccharides isolated from C. militaris in preventing obesity, we analyzed body weight, fat content, lipid gene, thermogenic protein and gut microbiota of mice using histopathology, q-PCR, Illumina Miseq (hypervariable V3 and V4 regions of the 16S ribosomal RNA gene). We hypothesized that (i) the high-yield CMP can reduce overweightness, fat accumulation, serum lipid index and modify gut microbiota community, thus remediate obesity; (ii) Se addition (SeCMP) can strengthen the effect of the CMP in body fat control; (iii) the intestinal microbiota community assembly in response to CMP compound result in favorable fat digestion/processing pathway. Artificial cultivated C. militaris is evidenced safe, widely available and abundant of polysaccharides, this work is valuable to for future therapeutic application and functional food development at large-scale.

\section{Materials and Methods}

\subsection{Materials and reagents}

C. militaris strain was isolated by Institution of Microbiology, Chinese Academy of Sciences (Beijing, China) from wild fruit bodies. Additional $0.04 \mathrm{mg} / \mathrm{g} \mathrm{Na} \mathrm{SeO}_{3}$ was mixed with C. militaris rice ferment medium. The product of C. militaris fruit body with/without Se were purchased from Nanguang Biotech develop Ltd, (Shanghai, China). C. militaris fruit body was identified by Bin Li (Associate research fellow, Chinese Academy of Sciences, Beijing, China). Chemical reagents were of analytical grade and purchased from commercial sources.

\subsection{Isolation of Crude Polysaccharide}

Crude polysaccharide of $C$. militaris (CMP) and Se-rich C. militaris (SeCMP) were prepared in a simple and quick method. The fruit bodies were dried and ground into powder. The extracts were prepared by mixing dried powder with deionized water $(1: 10, w / v)$ before ultrasonic concussion for $30 \mathrm{~min}$ at $50{ }^{\circ} \mathrm{C}$, and then boiled at $121^{\circ} \mathrm{C}$ for $30 \mathrm{~min}$. The solution was centrifugation at $4000 \mathrm{rpm}$ for $15 \mathrm{~min}$ at room temperature using Bioridge TD5M-WS centrifuge ( $\mathrm{Lu}$ Xiangyi Centrifuge Instrument Co., Ltd, Shanghai, China). The supernatants were concentrated to $1 / 4$ volume at $65^{\circ} \mathrm{C}$ using EYELA SB1100 vacuum concentrator (Tokyo, Japan). Three times absolute ethanol was added to the concentration and stored at $4{ }^{\circ} \mathrm{C}$ overnight. The precipitates were collected and redissolved in deionized water, concentrated and lyophilizated into powder using Virtis BenchTop Pro freeze dryer (USA). Crude polysaccharide [30], crude proteins [31], crude fat [32], hydrolyzed amino acids [33], total phenol [34, 35] and Se content [36] of SeCMP and CMP were measured according to the previous reported method with a little modification. Total diet fiber was determined using an enzymatic gravimetric method (AOAC Methods 2011.25). Moisture and ash content were determined using gravimetric method. Carbohydrate and energy were calculated from formulas

$$
\begin{gathered}
m \text { Carbohydrate }(\mathrm{g})=m_{\text {Total sample }}(\mathrm{g})-m_{\text {protein }}(\mathrm{g})-m_{\text {Moisture }}(\mathrm{g})-m_{\text {Ash }}(\mathrm{g})-m_{\text {Diet fiber }}(\mathrm{g}) \\
E(\mathrm{kcal})=4(\mathrm{kcal} / \mathrm{g}) \times m \text { Carbohydrate }(\mathrm{g})+9(\mathrm{kcal} / \mathrm{g}) \times m_{\text {Fat }}(\mathrm{g})+4(\mathrm{kcal} / \mathrm{g}) \times m_{\text {Protein }}(\mathrm{g})
\end{gathered}
$$

where $m$ is mass and $E$ is energy of each polysaccharides extracts.

\subsection{Animal Model and Experimental Design}

This study was approved by Institutional Animal Care and Use Committee of Shanghai Jiaotong University (IACUC; No. 2018089). A total of 32 four-week-old C57BL/6J male mice (specificpathogen-free grade) were purchased from the Beijing Vital River Laboratory Animal Technology Co., Ltd. (Beijing, China). The mice were randomly housed 4 individuals per cage with food and sterile drinking water ad libitum in a controlled environment (temperature at $26^{\circ} \mathrm{C} \pm 1{ }^{\circ} \mathrm{C}, 12$-hour darklight cycle). Normal diet (5.5\% of energy from fat) and high fat diet (60\% of energy from fat) were purchased from Xinhui animal Stall Food Co.,Ltd. (Shanghai, China). After 1-week accommodation 
period, mice were divided into 4 groups and fed with sterile water (vehicle) + normal diet (CHOW group, $n=8$ ); sterile water + HFD diet (HFD group, $n=8) ; 100 \mathrm{mg} / \mathrm{kg}$ bw/day CMP + HFD diet (CMP group, $n=8) ; 100 \mathrm{mg} / \mathrm{kg}$ bw/day SeCMP + HFD diet (SeCMP group, $n=8$ ) by intragastric gavage during the 8 weeks. The mice were weighed once per week throughout the experiment period. Food and water consumption of each cage was monitored twice a week and converted into daily intake per mouse. The stool lipid content was assessed by gravimetric analysis procedure as issued before [37]. At the end of animal study, fasting animals were anaesthetised with isoflurane and then the blood was collected by cardiac puncrure. Brown and white adipose fat (white adipose fat including subcutaneous, epididymal and perirenal fat) were carefully removed and weighed, along with liver, heart, spleen, lung, kidney and pancreas. Organs and tissues for staining were immersed in 10\% neutral buffered formalin solution (Sangon biotech, Shanghai, China) or liquid nitrogen and then stored at $-80^{\circ} \mathrm{C}$ for further analysis.

\subsection{Serum cytokines measurement}

Whole blood was collected by cardiac puncture in tubes without anticoagulant. It was allowed to clot for $30 \mathrm{~min}$ and then centrifuged at $2000 \mathrm{~g}$ for $10 \mathrm{~min}$, collecting supernatant (serum) for determination. Serum total cholesterol (TC), triglyceride (TC), high-density lipoprotein-cholesterol (HDL-C) and low-density lipoprotein-cholesterol (LDL-C), alanine aminotransferase (ALT), aspartate aminotransferase (AST), uric acid (UA), creatinine (CRE), albumin (ALB), total bilirubin (TBIL) were quantified using commercial kit produced by Nanjing Jiancheng Bioengineering institute (China). Leptin and adiponectin ELISA kit were purchased from Beyotime Biotechnology (Shanghai, China).

\subsection{Oil red $O$ staining}

Formalin fixed liver tissues were stained with Oil Red O (Servicebio, Wuhan, China) for 30 min, rinsing with $75 \%$ ethyl alcohol and then stained with haematoxylin for $2 \mathrm{~min}$. Sections were examined with the NIKON ECLIPSE TI-SR inverted fluorescence microscope (NIKON, Japan) and scanned sections were captured using NIKON DS-U3 microscope camera controller (NIKON, Japan). A total of 3 tissue sections were analyzed for each animal using image J (v1.8.0).

\subsection{Histopathological staining}

Epididymal white adipose tissues (eWATs) immersed in formalin beforehand were embedded with paraffin and then stained with hematoxylin and eosin (H\&E) for morphological examination. Slides were scanned and gene expression was quantified as mentioned in 2.5.

\subsection{Immunohistochemistry (IHC) staining}

IHC staining was performed on paraffin embedded eWATs and BATs sections hybridized with rabbit anti mouse uncoupling protein 1 antibody (UCP1, Abcam, UK) before exposed to 3,3diaminobenzidine (DAB). Cell nuclei were counterstained with hematoxylin. Slides were scanned and gene expression was quantified as mentioned in 2.5 .

\subsection{RNA Extraction and q-PCR Analysis}

Total RNA was extracted from liver and eWATs using TRIzol reagent (Invitrogen, CA, USA), which was then used to synthesize cDNA with PrimeScript RT reagent kit with gDNA Eraser (Takara, Japan). Q-PCR was performed using TB Green® Premix Ex Taq ${ }^{\text {TM }}$ II (Tli RNaseH Plus) (Takara, Japan) and Applied Biosystems StepOne Real-Time PCR instrument (Life Technologies, Singapore). Relative amounts of target genes were normalized to the housekeeping gene GAPDH and calculated based on the $2^{-\Delta \Delta \mathrm{Ct}}$ method. Genes and primer sequences were listed in Table S5. 


\subsection{Determination of stool short chain fatty acids (SCFAs)}

Stool were reconstituted in deionized water, then acidified the supernatants with $50 \% \mathrm{H}_{2} \mathrm{SO}_{4}$ and SCFAs were extracted by ethyl ether. Quantitative analysis of stool acetate, propionate, butyrate, valeric acid, isobutyric acid and isovaleric acid were measured on gas chromatography-triple quadrupole mass spectrometry (7890B-7000D) with a FFAP capillary column $(30 \mathrm{~m} \times 0.25 \mathrm{~mm} \times 0.25$ $\mu \mathrm{m})$ (Agilent Technologies, CA, USA). Carrier gas was $1 \mathrm{~mL} / \mathrm{min}$ helium. The injector temperature was $250{ }^{\circ} \mathrm{C}$. The oven temperature was set at $100^{\circ} \mathrm{C}$ maintained for $1 \mathrm{~min}$, raised to $145^{\circ} \mathrm{C}$ at $5{ }^{\circ} \mathrm{C}$ $/ \mathrm{min}$, increased to $240{ }^{\circ} \mathrm{C}$ at $15{ }^{\circ} \mathrm{C} / \mathrm{min}$, maintained for $8 \mathrm{~min}$. Ion source temperature: $230{ }^{\circ} \mathrm{C}$; ionization mode: EI+, $70 \mathrm{eV}$; scanning mode: selected ion monitor (SIM). The contents of stool SCFAs were calculated with external standard methods.

\subsection{Cecal microbiota DNA extraction}

Faeces samples were snap-frozen in liquid nitrogen after separation from cecal and stored at $80^{\circ} \mathrm{C}$. Total bacterial genomic DNA were extracted using the Mag-Bind ${ }^{\circledR}$ DNA Kit (QIAGEN, Inc., Netherlands). The quality and quantity of the extracted DNAs were measured using a NanoDrop ND-1000 spectrophotometer (Thermo Fisher Scientific, Waltham, MA, USA) and agarose gel electrophoresis, respectively. Then, the V3-V4 regions of $16 \mathrm{~S}$ rRNA were amplified using composite primers containing the forward primer 338F (5'-ACTCCTACGGGAGGCAGCA-3') and the reverse primer 806R (5'-GGACTACHVGGGTWTCTAAT-3'). The PCR amplicons were purified using Agencourt AMPure Breads (Beckman Coulter, Indianpolis, IN), then quantified with the PicoGreen dsDNA Assay Kit (Invitrogen, Garlsbad, CA, USA). The amplicons were pooled into equal amounts after the individual quantification step and paired-end $2 \times 300 \mathrm{bp}$ sequencing was performed on an Illumina MiSeq platform with MiSeq Reagent Kit V3 at Shanghai personal Biotechnology Co., Ltd (Shanghai, China). The sequence data were submitted to NCBI sequence read archive (SRA) (accession number: SRR12927607-SRR12927629).

\subsection{Bioinformatics and statistics}

Sequence data analyses were performed with QIIME 2 software (2019.4) with slight modification according to the official tutorials. Briefly, raw sequence data were demultiplexed following by primers cutting. Sequences were then qualified for high-quality reads through filtering, denoising, merging and chimera removing process using the DADA2 plugin. Non-singleton amplicon sequence variants (ASVs) were aligned and used to construct a phylogeny. To avoid sampling depth bias, the abundance of ASVs are randomly rarefied from each sample to reach a uniform depth (at $95 \%$ of the smallest samples amounts) (Qiime feature-table rarefy). Alpha-diversity metrics Observed species, Simpson [38] and Pielou's evenness [39] indexes were calculated using QIIME2. Beta diversity analysis was performed to using bray-curtis distance based nonmetric multidimensional scaling (NMDS) and unweighted pair-group method with arithmetic means (UPGMA) hierarchical clustering [40] based on Bray-curtis distance were calculated using the diversity plugin in QIIME2. The significance of differentiation of microbiota structure among groups was assessed by PERMANOVA (Permutational multivariate analysis of variance) and ANOSIM (Analysis of similarities) [41] using R software. Taxonomy was assigned to ASVs using the classify-sklearn naïve Bayes taxonomy classifier in feature-classifier plugin [42] against the Greengenes 13_8 [43] 99\% OTUs reference sequences. Taxa abundances at the phylum, class, order, family, genus and species levels were statistically compared between groups by multivariate statistical analysis. LEfSe (Linear discriminant analysis effect size) was performed to detect differentially abundant taxa across groups using Python LEfSe package, R software [44].

The chemical content in CMP and SeCMP were compared using two-tailed independent student $t$ test. Data among groups were assessed using one-way analysis of variance (ANOVA) followed by Tukey's post hoc test or the non-parametric Kruskal-Wallis test with Dunnett's T3 multiple 
comparison test by GraphPad Prism 8.0 (GraphPad Software, USA). Data are presented as mean \pm standard error (SE). Correlation coefficients between bacterial species and obesity traits were determined using Pearson's correlation analysis with Origin 2021. $P$ values $<0.05$ were considered to be statistically significant.

\section{Results}

\subsection{Effects of CMP on physiological indicators in HFD-induced obese mice}

CMP and SeCMP were obtained at the rate of $14.3 \pm 0.7 \%$ and $15.2 \pm 0.9 \%$, respectively, suggesting these extracts were high-yield compared with purity polysaccharides [45] and organic solvent extraction [20]. Table 1 shows chemical characteristics of the CMP and SeCMP samples in this study. Polysaccharides content is an important indicator for this assay. High content of crude polysaccharide and carbohydrate in CMP and SeCMP, implying the extracts were abundant in sugars. The Se content in Se-deficient C. militaris fruit body was much lower than in Se-rich $C$. militaris at $0.1 \pm 0.0 \mathrm{mg} / \mathrm{kg}$ and $3.9 \pm 0.6 \mathrm{mg} / \mathrm{kg}(p<0.001)$, while Se content in SeCMP was 50 times higher than in CMP, indicating the Se yield ratio was not that high in water extract fraction. Apart from Se and polysaccharides content, protein also present the major component in extracts. Minor compounds included diet fiber, phenol, and fat. Although a few discrepancies in compounds presented between the two extracts, these might also be potential contributor to differing biological action.

Table 1. Chemical characterization of CMP and SeCMP (Per $100 \mathrm{~g}$ dried sample)

\begin{tabular}{lll}
\hline & CMP & SeCMP \\
\hline Se $^{*}$ & $0.1 \pm 0.0 \mathrm{mg} / \mathrm{kg}$ & $5.1 \pm 0.2 \mathrm{mg} / \mathrm{kg}$ \\
Diet fiber $^{*}$ & $12.4 \pm 0.3$ & $8.8 \pm 0.2$ \\
Carbohydrate $^{*}$ & $37.8 \pm 0.2$ & $45.0 \pm 0.3$ \\
Crude polysaccharide $^{*}$ & $47.4 \pm 2.0 \%$ & $56.7 \pm 3.9 \%$ \\
Protein $^{*}$ & $35.1 \pm 0.0$ & $31.1 \pm 0.2$ \\
Fat $^{*}$ & $1.2 \pm 0.2$ & $1.3 \pm 0.2$ \\
Hydrolyzed amino acids $^{*}$ & $20.8 \pm 0.4$ & $18.5 \pm 0.3$ \\
Phenol $^{*}$ & $2.1 \pm 0.0$ & $1.7 \pm 0.0$ \\
Energy $^{*}$ & $302.2 \pm 0.8 \mathrm{kcal}$ & $316.3 \pm 1.3 \mathrm{kcal}$ \\
\hline
\end{tabular}

Data are presented as mean \pm SE $(n=3)$. Data were analyzed by two-tailed independent student $t$ test using GraphPad Prism 8.0. ${ }^{*}$ Indicated significant variation between groups; ${ }^{*} p<0.05$.

Daily administration of CMP and SeCMP did not affect mean food intake, water intake and stool fat excretion (Figure S1), indicating that body weight and tissue fat accumulation regulation effect might derive from CMP and SeCMP induced internal physiological modulation instead of affecting appetite, food digestion or lipid extraction. Body weight (Figure 1a-c) and fat accretion in fat depots, including perirenal, epididymal and subcutaneous fat pad (Table 2) of mice fed with HFD were remarkably increased than the control CHOW group mice $(p<0.0001)$. These symptoms were not affected by CMP administration (Figure 1a-c). Diminished adipocytes size was observed in CMPtreated mice versus HFD-treated group $(p<0.01)$ (Figure 1d). Diet-induced hepatic steatosis was fully inhibited by CMP treatment contrast to HFD group $(p<0.01)$, as indicated by quantitative result of total hepatocytes lipids using Oil Red O staining assay (Figure 1e). However, these parameters were not improved by SeCMP intervention versus CMP group. Taken together, these results indicated that CMP ameliorated HFD-induced obesity mice adipocyte and liver morphological phenotype rather than body weight gain, while Se addition showed no significant promotion compared with simply CMP remedy. 

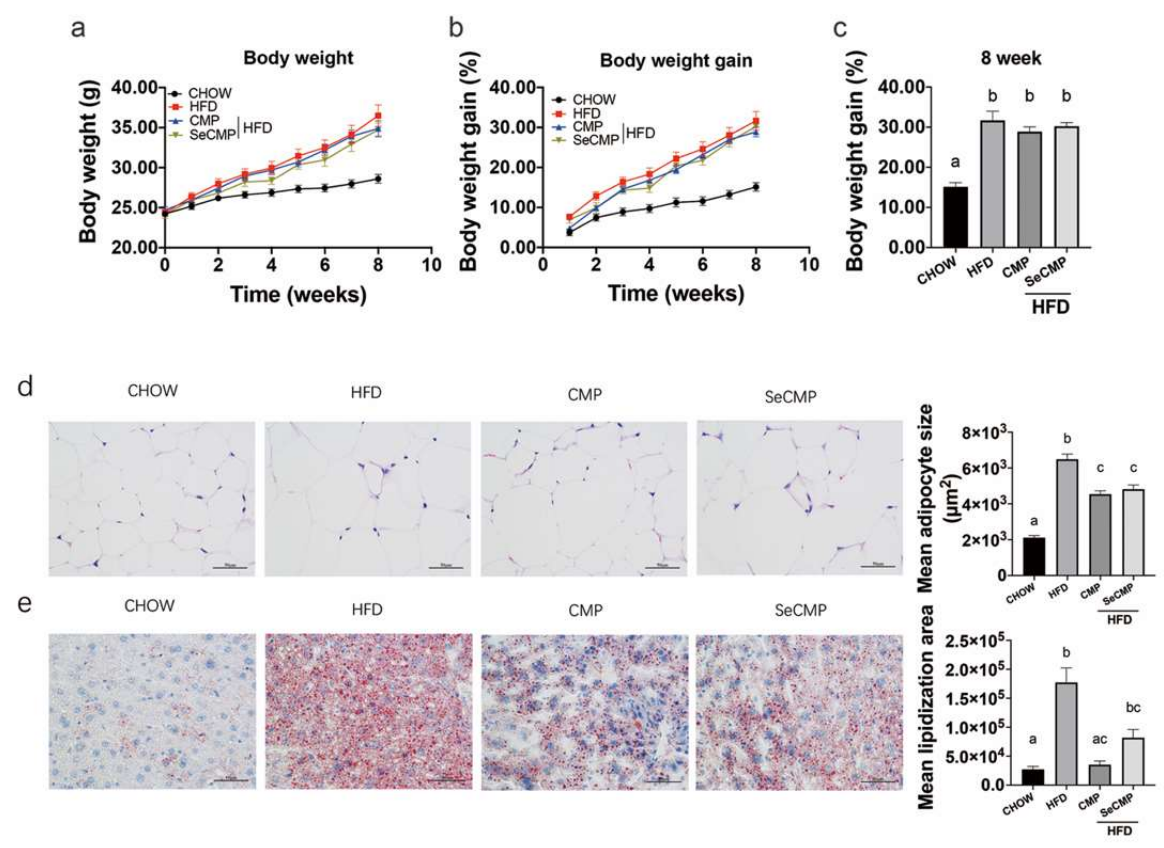

Figure 1. The effect of CMP and SeCMP on diet-induced obesity in mice. Mice were treated daily with CHOW, HFD, HFD+CMP (100 mg/kg) and HFD+SeCMP $(100 \mathrm{mg} / \mathrm{kg})$ by intragastric gavage for 8 weeks. The body weight (a), body weight gain (b) throughout the experiment and body weight gain at the 8 th week (c) are shown. Data are expressed as mean \pm SE ( $n=8$ for each group). Data were analyzed using one-way ANOVA analysis with Tukey's post hoc test or the non-parametric KruskalWallis test with Dunnett's T3 multiple comparison test. Superscript characters indicate significant variation among treatments.

Table 2. Organs and tissues weight (g)

\begin{tabular}{lccccc}
\hline & Liver & Perirenal fat & $\begin{array}{c}\text { Epididymal } \\
\text { fat }\end{array}$ & $\begin{array}{c}\text { Subcutaneous } \\
\text { fat }\end{array}$ & $\begin{array}{c}\text { Brown adipose } \\
\text { tissues }\end{array}$ \\
\hline CHOW & $1.041 \pm 0.021^{\mathrm{a}}$ & $0.120 \pm 0.019^{\mathrm{a}}$ & $0.425 \pm 0.047^{\mathrm{a}}$ & $0.235 \pm 0.028^{\mathrm{a}}$ & $0.129 \pm 0.010^{\mathrm{a}}$ \\
HFD & $0.832 \pm 0.016^{\mathrm{b}}$ & $0.471 \pm 0.043^{\mathrm{b}}$ & $1.221 \pm 0.090^{\mathrm{b}}$ & $0.703 \pm 0.070^{\mathrm{b}}$ & $0.138 \pm 0.007^{\mathrm{a}}$ \\
CMP & $0.821 \pm 0.021^{\mathrm{b}}$ & $0.430 \pm 0.049^{\mathrm{b}}$ & $1.110 \pm 0.079^{\mathrm{b}}$ & $0.617 \pm 0.060^{\mathrm{b}}$ & $0.122 \pm 0.007^{\mathrm{a}}$ \\
SeCMP & $0.755 \pm 0.021^{\mathrm{b}}$ & $0.499 \pm 0.054^{\mathrm{b}}$ & $1.231 \pm 0.076^{\mathrm{b}}$ & $0.722 \pm 0.066^{\mathrm{b}}$ & $0.130 \pm 0.009^{\mathrm{a}}$ \\
\hline
\end{tabular}

Data are expressed as mean \pm SE ( $n=8$ for each group). Data were analyzed using one-way ANOVA analysis with Tukey's post hoc test or the non-parametric Kruskal-Wallis test with Dunnett's T3 multiple comparison test. Superscript characters indicate significant variation among treatments.

The HFD dramatically elevated serum lipid parameters, including serum cholesterol $(p<0.0001)$, triglyceride $(p<0.05)$, HDL-C $(p<0.0001)$ and LDL-C $(p<0.0001)$ compared with CHOW-fed mice (Figure 2a-d). No profound decrease was observed between HFD and CMP group, whereas SeCMP treatment fully prevented HFD increased serum triglyceride level compared with CMP $(p<0.05)$. Serum LDL-C level was slightly reduced by SeCMP treatment versus HFD-fed mice $(p=0.095)$, yet not significant. Tissues weight were not affected by CMP and SeCMP intervention (Table S1), as well as, serum ALT, AST, UA, CRE, T-BIL and ALB parameters (Table S2), indicating that CMP and SeCMP did not cause liver and kidney function damage. In summary, CMP was not a good 
hyperlipidemia alleviator, however, SeCMP intervention blunted dyslipidemia more notably than CMP, as indicated by lower serum triglyceride and LDL-C response.
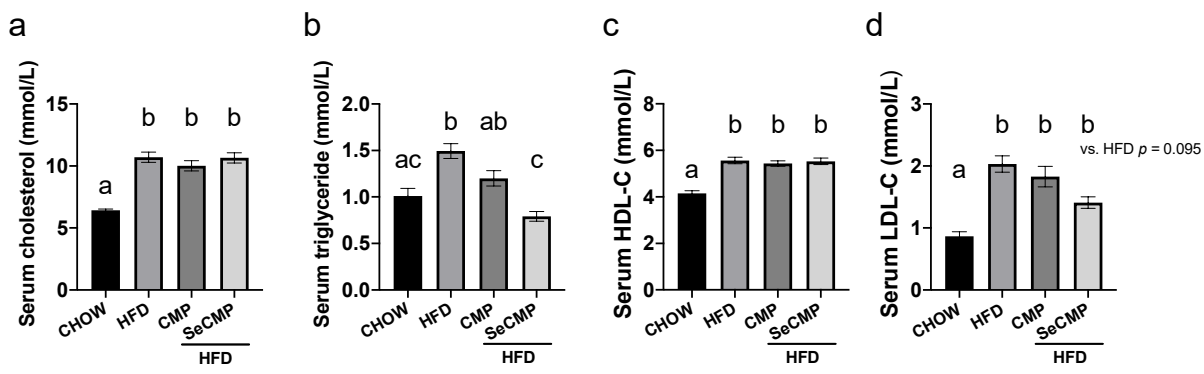

e

Liver lipid
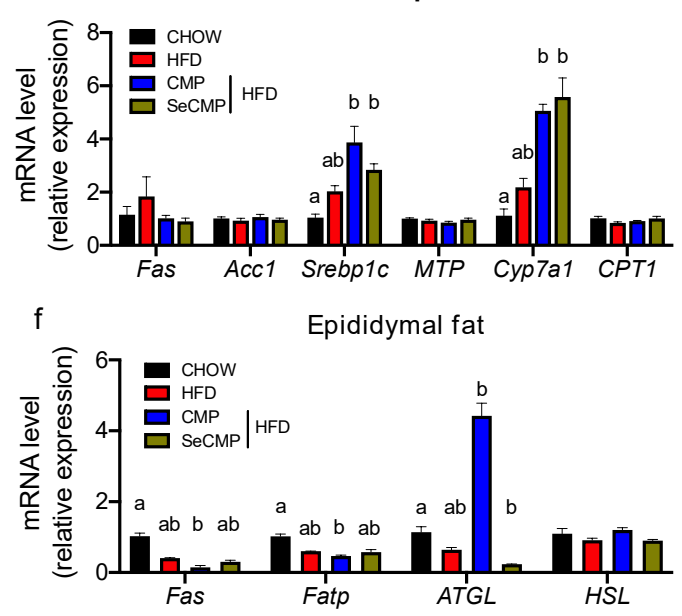

Figure 2. The modulation effect of CMP and SeCMP on dyslipidemia in obesity mice. Serum (a) cholesterol, (b) triglycerides, (c) HDL-C and (d) LDL-C ( $n=8$ for each group) were shown. Q-PCR analysis was performed to quantify lipid absorption, distribution, and metabolism genes expression in (e) liver and (e) hepatic tissues. The genes expression involved lipogenesis (fatty acid synthase, Fas; acetyl-CoA carboxylase, ACC1; sterol regulatory-element binding protein 1c, Srebp1c), lipid transport (microsomal triglyceride transfer protein, MTP; fatty acid transport protein, Fatp), lipolysis (hormone-sensitive lipase, HSL; adipose triglyceride lipase, ATGL; cholesterol $7 \alpha$-hydroxylase, Cyp7a1) or $\beta$-oxidation (carnitine palmitoyltransferase $1, C P T 1)$. Data represent mean \pm SE $(n=6$ for each group). Data were analyzed using one-way ANOVA followed by Tukey's post hoc test or the non-parametric Kruskal-Wallis test with Dunnett's T3 multiple comparison test. Superscript characters indicate significant variation among treatments.

\subsection{Mechanism of CMP and SeCMP supplementary on dyslipidemia in HFD-induced obese mice}

We determined genes expression involved in lipogenesis, transport and lipolysis and $\beta$ oxidation in hepatic and adipose tissues to elucidate the underlying mechanism of CMP and SeCMP on lipid absorption and metabolism (Figure 2e, f). Compared with CHOW mice, HFD intervention did not affect gene expression tested in this study. The expression of gene Srebp1c $(p<0.01)$ and Cyp7a1 $(p<0.01)$ relating to lipid synthesis and BAs synthesis pathways were notably increased by $\mathrm{CMP}$ treatment in liver, respectively, versus $\mathrm{CHOW}$ group, implying the modulating dyslipidemia effect of CMP in HFD model mice might associate with accelerating cholesterol transform into BAs, which was also observed in SeCMP administration mice.

BAT is dense capillary nurture adipocytes in mice providing source of heat against cold exposure and enabling distribution of heat to the rest of body [46]. We determined expression of 
mitochondrial UCP1, which was identified before as a major regulator of thermogenesis in adipose tissues. Both CMP and SeCMP administration improved morphological changes by HFD. Notably, the expression of UCP1 protein in BAT was remarkably suppressed by HFD supplement $(p<0.0001)$, but no difference was observed between CMP and HFD group. SeCMP showed no difference versus CMP group, but increased UCP1 expression compared with HFD group $(p<0.05)$ (Figure 3), suggesting SeCMP restrained fat accumulation in tissues might through improving BAT thermogenesis in HFD-fed mice.

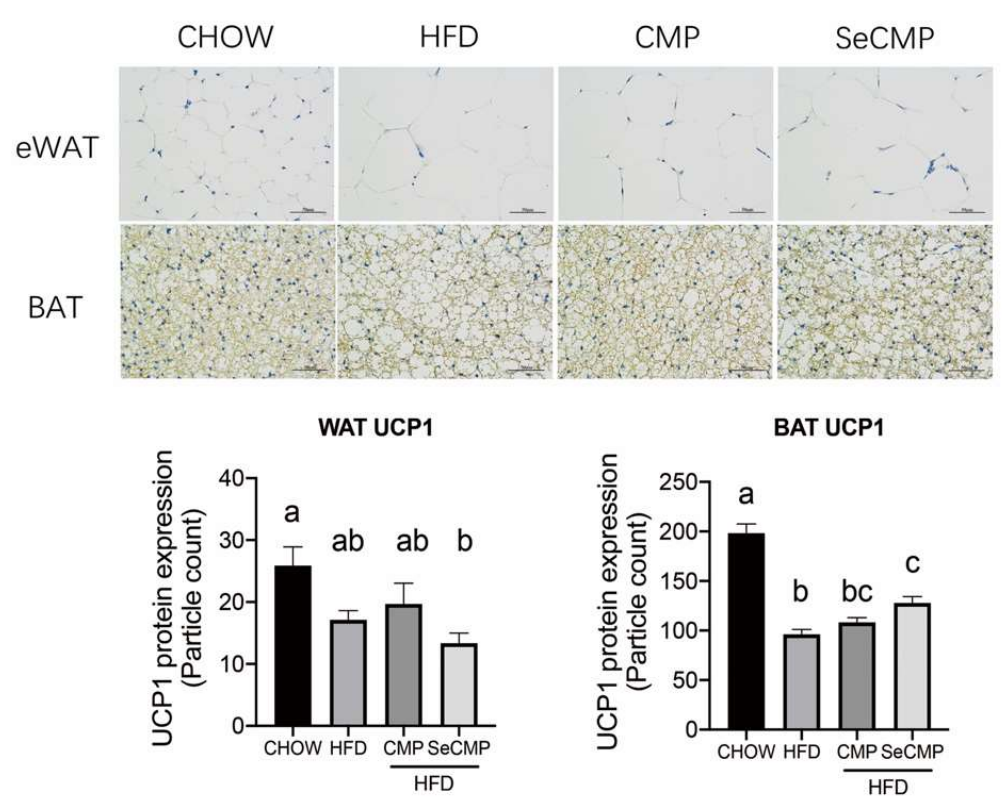

Figure 3. CMP and SeCMP induce expression of thermogenic markers in HFD fed mice. Representative images of UCP1-stained epididymal visceral adipose tissue (eWAT) and BAT. Scale bars, $50 \mu \mathrm{m}$. UCP1 protein expression was quantified using image J. Data represent mean \pm SE $(n=6-$ 8 mice for each group). Data were analyzed using one-way ANOVA followed by Tukey's post hoc test or the non-parametric Kruskal-Wallis test with Dunnett's T3 multiple comparison test. Superscript characters indicate significant variation among treatments.

Previous study has evidenced that fiber and polysaccharide fermentation restored the abundance of health-beneficial microbes and increased liner-chain SCFAs production, such as acetate acid, propionate acid, and butyrate acid to promote body weight loss, while gut microbes fermented with protein and fat enriched diet produced branched-chain SCFAs metabolites such as isobutyrate acid, isovalerate acid and 2-methylbutyrate acid, which were reported associating with insulin resistance [47, 48]. Hence, gut microbes favored polysaccharides for growth from diet and produced beneficial liner-chain SCFAs for modulating gut dysbiosis and in turn enhanced human health. In this work, CMP and SeCMP did not affect caecal production of acetic acid, propionic acid, butyric acid, isobutyric acid, valeric acid and isovaleric acid (Figure S2).

\subsection{Effects of CMP on gut microbiota dysbiosis in HFD-driven obese mice}

Gut microbiota diversity was described by richness, diversity and evenness indexes to evaluate the effect of polysaccharide intervention on obesity mice models. Bacterial richness (Observed species) was reduced in HFD mice gut compared with CHOW-fed mice $(p<0.05)$, CMP showed no effectual action on increasing microbial richness. Simpson and Pielou's evenness indeses were not 
difference between HFD and CHOW groups, however, these parameters were significantly decreased after CMP treatment $(p<0.05)$. These results implied the overall gut microbial diversity in mice fed with HFD could not be improved by CMP intervention. Bray-curtis distance based nonmetric multidimensional scaling (NMDS) revealed a distinct clustering of bacterial composition between HFD group and CHOW group. CMP group showed significant separation to HFD group, indicating that CMP intervention alter the composition of gut bacteria in the HFD-fed mice (Figure 4a). These findings were verified by Bray-curtis distance based UPGMA hierarchical clustering (Figure S3a). Pair-wise PERMANOVA and ANOSIM tests indicated a statistically significant separation between groups ( $p<0.05$ ), supporting the NMDS result (Table S3 and Table S4). The alpha and beta diversity trend of SeCMP group coincided with that of CMP group. The above results indicated that CMP and SeCMP relieved dyslipidemia might via altering the composition in the gut microbiota.
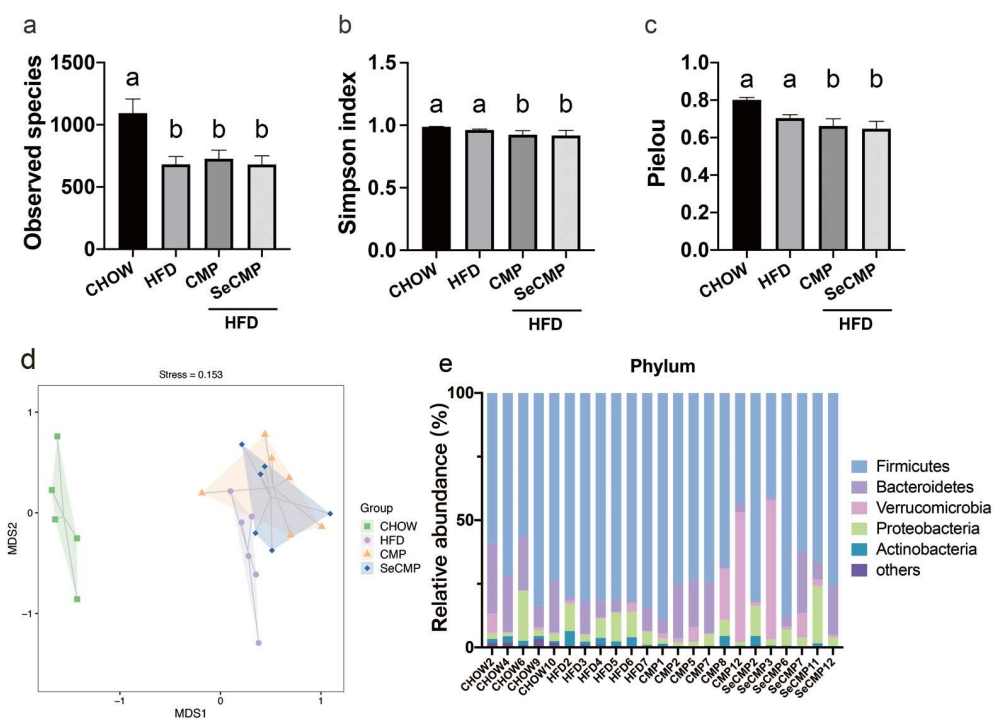

Figure 4. CMP and SeCMP do not affect richness, diversity and evenness of gut microbiota, as well as bacteria at phylum and class level. Alpha diversity indexes of observed species, Simpson and Pielou's evenness index is represented $(\mathrm{a}-\mathrm{c})$. Bray-curtis distance based nonmetric multidimensional scaling (NMDS) (d) was performed to investigate the structural variation of microbial communities across samples. (e) Bacterial taxonomic profiling in the phylum level of bacteria. (f) The relative abundance of the profound altered bacteria at order level. Data represent mean \pm SE $(n=5-6$ mice for each group). Data were analyzed using Tukey's post hoc test or the non-parametric Kruskal-Wallis test with Dunnett's T3 multiple comparison test. Superscript characters indicate significant variation among treatments.

We used multivariate statistical analysis to identify the indicator microbes in each group. In accordance with the previous studies the mouse microbiome was greatly dominated by the phyla 
Firmicutes (72.8\%) and Bacteroidetes (11.3\%) [49] (Figure 4b). We observed no significant difference between treatments on gut bacterial populations at the phylum and class level. At the order level, we found greater relative abundance of Lactobacillales in HFD group (Figure 4h), but was lower in CMP $(\mathrm{p}<0.05)$. At the genus level (Figure 5 and supplementary figure 4$)$, the abundance of Lactobacillus, Dorea, Clostridium, Streptococcus and Christensenella significantly increased in HFD-induced hyperlipidemic mice $(\mathrm{p}<0.05)$ were prominently reversed by CMP intervention. In addition, LEfSe approach ranked above mentioned genus as the main features of HFD group mice. In conclusion, we decided to focus on Lactobacillus, Dorea, Clostridium and Streptococcus as markers stimulated by CMP.

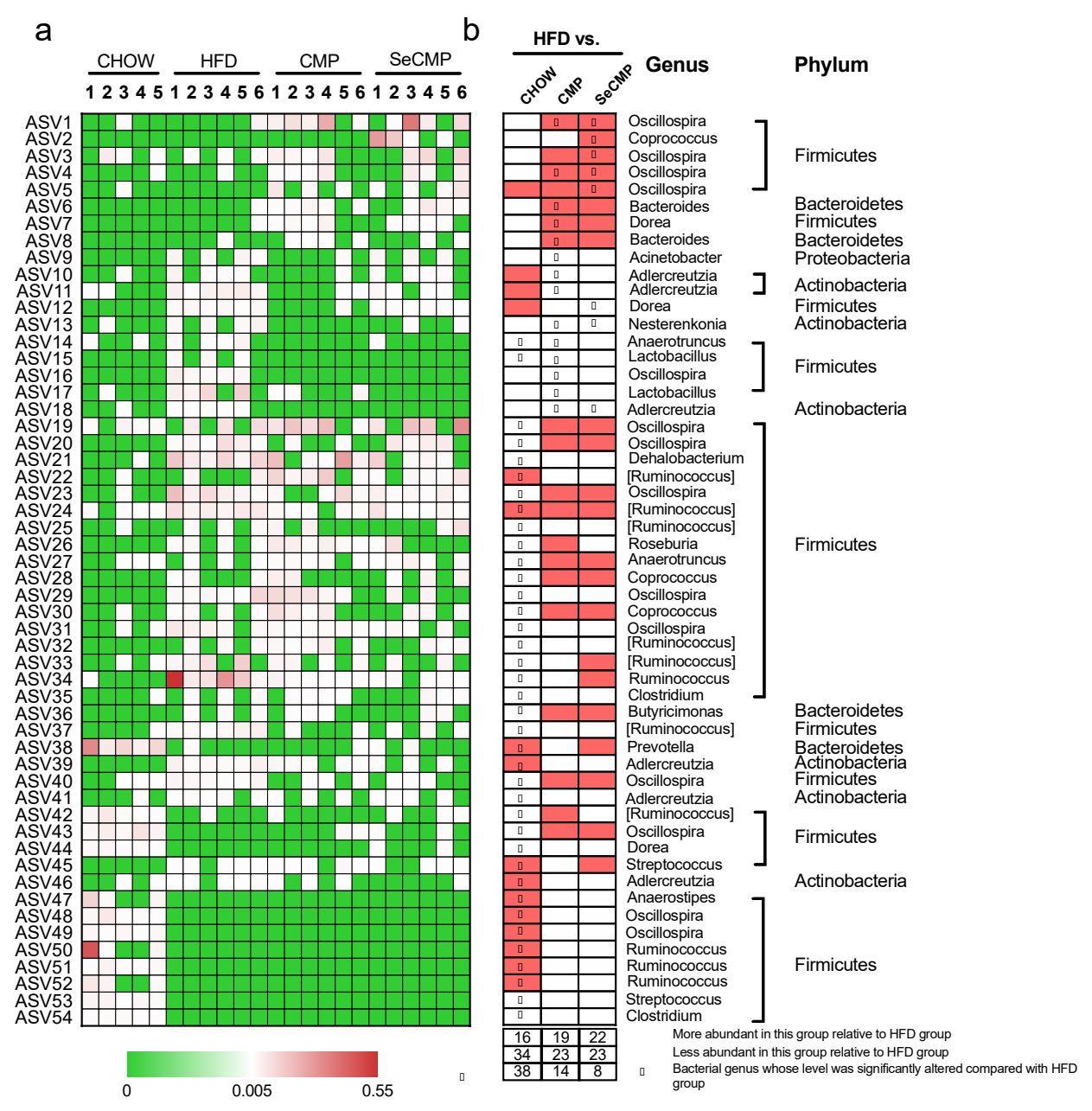

Figure 5. CMP and SeCMP affect specific bacteria at genus level. (a) Heatmap showing the levels of 54 bacterial genera significantly altered by CHOW, CMP and SeCMP intervention in comparison with the HFD group based on MetagenomeSeq analysis. (b) Bacterial genera from panel (a) and changes induced by the treatments indicated. Red and green blocks indicated genera that were more and less abundant in the groups compared with HFD group. Black stars indicated genera levels were significantly altered by HFD but reversed by treatments.

The Pearson's correlation analysis was used to clarify the correlation of relative abundance top 10 genera and indicator genera (Figure 6) with obesity indexes. The CMP and SeCMP groups depleted genus Lactobacillus, Dorea, Clostridium and Coprobacillus were positive associated with the lipid metabolism-related parameters, especially LDL-C level $(p<0.05)$, suggesting that they may be the most important genera for the development of hyperlipidemia. Notably, Ruminococcus showed a 
slight decrease tendency under CMP intervention revealed positive association with obesity-related serum lipid indexes $(p<0.05)$, indicating that it might also play a role in improving fat accumulation and dyslipidemia. In conclusion, CMP intervention decreased specific obesity-relating gut microbes in HFD fed obese mice.

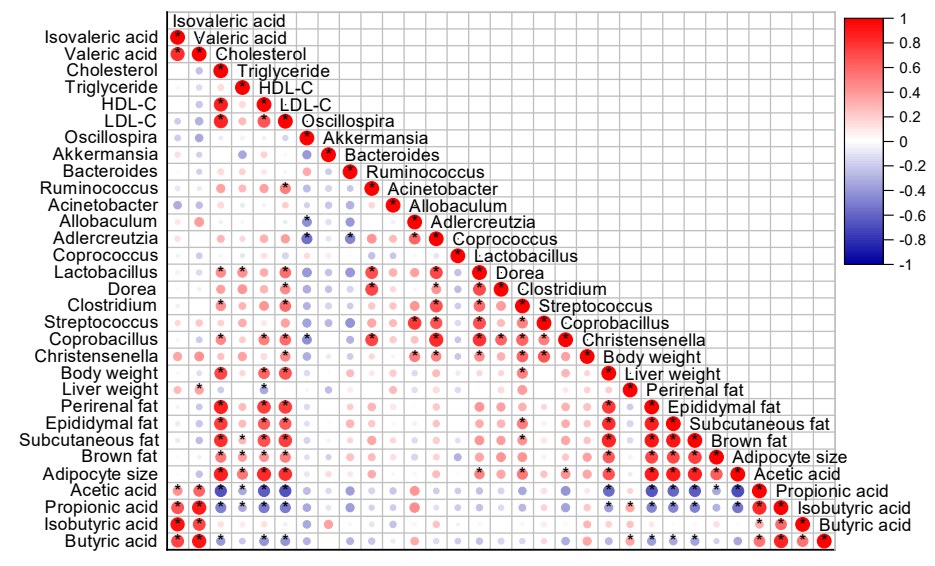

Figure 6. CMP and SeCMP supplementary reduce pernicious bacteria positively correlate with obesity traits. Correlation matrix between the indicator genera (relative abundance top 10 genera and identified in multivariate statistical analysis) and obesity traits using Pearson's correlation analysis. ${ }^{*} p<0.05$.

\section{Discussion}

\subsection{Intervention of CMP on fat accumulation and hyperlipidemia}

Substances from cultured C. militaris have been proved safe and excellent alternations for obesity prevention [50] because water-soluble fraction overcome the disadvantage of low yield rate, sever side effect and pollution issues of the small organic molecule. Here we obtained high-yield crude polysaccharides using a simple and eco-friendly method with yield ratio at about $15 \%$, which was much higher than extract from C. militaris isolated with 50\% ethanol (6.2\%) [20], because of the high content of polysaccharide and various water-soluble nutritions in the extracts. The organic constituents can reduce body weight remarkably at $100-300 \mathrm{mg} / \mathrm{kg}$ bw fed with $27 \%$ fat in diet, which was different from our results, this might be due to the intolerance caused by the rather high-in-fat feeding $(60 \%)$ and low test dosage adopted in this work. Administration of CMP proved decreasing fat accumulate in liver and adipose and hypertriglyceridemia effect, which was consistent with previous findings $[6,20]$, indicating the prospective application as obesity remedy. These findings support our first hypothesis CMP can reduce obesity-induced fat accumulation in obesity murine. $C$. militaris can be a candidate developed as functional food due to the simple preparation procedure and culturally available trait.

To identify the ingredients that actually work, compounds and nutrients in CMP and SeCMP were characterized, suggesting polysaccharide composed the majority of the extracts (about 50\%). Although polysaccharides are predominantly enriched in the extracts, we could not rule out the possibility that other bioactive compounds present in the extract may also contribute to normalizing hyperlipidemia effects. It is widely acknowledged pharmacological activity is largely determined by molecular weight of polysaccharide [6,51]. Nevertheless, which fraction regulated the lipid metabolism of obese mice in our study is still not clear. CSP from C. sinensis purified with DEAE-52 
chromatography was capable of relieve obesity at $300 \mathrm{mg} / \mathrm{kg}$ bw [22]. However, although molecular weight is an important factor affecting biological activities, to obtain pure polysaccharide is a timeconsuming, high-cost and complicated procedure, and it is not always necessarily beneficial for pharmacological effect promotion. Thus, application of crude extracts as functional food tended to be more practical than pure polysaccharides.

Se-based products are widely marketed for their alleged health-promotion effect via strong antioxidant and anti-inflammatory potential [52]. Previous studies have generally shown some Seprebiotics supplementation; for instance, Se fermented probiotics complex [27], Se modified polysaccharide [28] and Se-rich product [29] were recognized to improve lipid metabolism and enhanced the activities of antioxidant enzymes of hyperlipidemic mice. What about Se-rich crude polysaccharide? We showed that mice treated daily with the same dose of SeCMP at $100 \mathrm{mg} / \mathrm{kg}$ bw (Se content is $5.1 \mathrm{mg} / \mathrm{kg}$ ) did not display stronger body weight gain and fat accumulation effect compared with CMP-fed mice, which is consistent with previous study [29]. Although Se content was high enough to be adsorbed on C. militaris fruit body, it was much lower than assembled with bacteria or pure polysaccharide, that result in poor effect. Hence, we assert a higher level of Se concentration is a necessity for biological activities and the vital of Se in obesity treatment has been exaggerated to some extent.

Obesity is associated with a series of serum lipid metabolic abnormalities characterized by hyper serum TG, TC, LDL-C and lower HDL-C. TG, TC and LDL-C are risk factors for cardiovascular disease such as arteriosclerosis $[53,54]$. HDL-C particles play a vital role in protecting against arterial disease due to their ability to promote cholesterol efflux from macrophages in the artery wall [55]. Previous studies had observed negative correlation between polysaccharide and serum TG, TC and LDL-C levels $[5,11]$. Our result was in agreement with previous study, who reported that serum lipid TC and LDL-C were sensitive to Se-rich complex supplementation in diet, implying SeCMP was more effective in improving lipid homeostasis than CMP. Nowadays, metabolically health is a new strategy to evaluate the degree of obesity in supplementary of the body mass index (BMI). Accumulating evidence [56] suggests that, although the risk of all-cause mortality and cardiovascular events might be higher in people with metabolically healthy obesity compared with metabolically healthy people of a normal weight, whereas, the risk is substantially lower than in individuals with metabolically unhealthy obesity $[57,58]$. Therefore, SeCMP supplementation is potential benefit to metabolically unhealthy individuals because of its profound ability to relieve murine of hypertriglyceridemia.

\subsection{CMP alleviate the HFD-induced obesity via modifying gut dysbiosis.}

Previous studies showed that the preventiveness of polysaccharides on obesity was closely associated with the restoration of intestinal microbiota [59-61]. Diets supplemented with sodium selenite positively stimulates mice gut bacteria diversity compared with Se-deficient diet in HFD fed mice [62]. We observed no significant difference in bacterial diversity between CMP and HFD supplementation. This outcome appears counterintuitive given the commonly accepted association between a lower gut bacterial diversity and poor health [21]. Nevertheless, previous studies have also found that dietary supplementation of a single fermentable substrate can reduce gut bacterial diversity, yet improve metabolic responses [63].

Multivariate statistical analysis of caecum bacteria samples revealed dietary intervention modified gut bacterial composition by reversing the HFD-induced flora dysbiosis. CMP altered the gut microbiome by profoundly reducing the genus Lactobacillus which was strongly correlated with serum cholesterol, triglyceride and LDL-C level. Lactobacillus is known to express bile salt hydrolases (BSH) result in the accumulation of intestinal conjugated bile acid [64]. Earlier study proposed that tempol increased conjugated bile acid (T- $\beta-\mathrm{MCA})$, then downregulated FXR in germ-free mice that showed a lean phenotype in mice, indicating Lactobacillus triggered anti-obesity effect probably by inhibiting FXR-mediate signaling in the intestine [65]. In our study, the upregulated CPY7a1 gene expression by CMP is a signal that the mechanism probably relating to BAs metabolism. Hence, further study is warranted to determine correlation between gut microbiota community and BAs metabolism pathway in remodeling lipid disorder. 
Among the members of gut microbiota altered by CMP, the majority of microbes were positively correlated with host lipid metabolism parameters, which were enriched by HFD but reduced by CMP. Previous studies showed that some Clostridium microbes might produce toxins. Chitosan administration increased the anti-obesity-related species Clostridium leptum, and significantly decreased Clostridium lactatefermentans and Clostridium cocleatum [66]. Ruminococcus and Dorea were common in inflammatory bowel disease or colitic individuals which were strongly correlated with inflammatory cytokine, such as TNF- $a$, these microbes might act as proinflammatory factors aggravating obesity progress $[67,68]$. Most of genus Streptococcus were conditioned pathogen and were associated with the development of multiple metabolic disorders IBD by promoting inflammatory cytokines [69]. The relative abundance of a series pathogen Clostridium, Ruminococcus, Dorea and Streptococcus were decreased in our study, unraveling CMP alleviated HFD-induced lipid disorder effect probably relating to reducing translocation inflammation cytokines from harmful bacteria.

\section{Conclusions}

We obtained two high-yield crude polysaccharide CMP and SeCMP using a simple and quick method, which ameliorated fat accretion in obese mice and modified gut microbiota dysbiosis. In addition, SeCMP exhibited superior protective effect against hyper triglyceride than CMP. In the future, study on large experimental animals or clinical trials were recommended to elucidate effect and mechanism relating to lipid regulation and therapeutic potential of obesity. In summary, this work provided theoretical basis for CMP and SeCMP in treating obese-induced fat accumulation and hyperlipidemia and successful development of CMP and SeCMP into functional food.

Supplementary Materials: Figure S1: Polysaccharides supplementation do not affect energy intake, water intake and energy extraction, Figure S2: CMP and SeCMP do not affect production of short chain fatty acids, Figure S3: The effect of CMP and SeCMP on gut bacteria beta diversity and the relative abundance at phylum level, Figure S4: The relative abundance of gut microbiota at genus level, Table S1: Organs weight, Table S2: Biochemistry analysis of serum of mice, Table S3: Group significance using ANOSIM analysis based on Bray-Curtis distance, Table S4: Group significance by PERMANOVA analysis based on Bray-Curtis distance; Table S5: Q-RCP primer sequences; Supplementary dataset (Microsoft Excel file): Relative abundance of bacterial species showing differences between the HFD-treated group and CHOW/CMP/SeCMP-intervented groups after feeding for 8 weeks (related to figure 5).

Author Contributions: P.Z., K.H., S.C. and D.Z. revised the final version. N.H. and X.Y. conceived the topic and revised the manuscript. M.Y. designed and conducted the experiment, and wrote the manuscript. J.W., Y.C., J.D. and X.C. conducted the sample collection and some determination. All authors have read and agreed to the published version of the manuscript.

Funding: This research was supported by grants from the Shanghai Agriculture Applied Technology Development Program, China (Grant No. T20180413) and Shanghai Science and Technology Innovation Action Project (Grant No.20392001000, 20dz1204804).

Acknowledgments: We thank instrumental analysis center of School of Agriculture and Biology (Shanghai Jiao Tong University) for chemical composition measurement. We thank the guidance and help for animal raising provided by animal center of Shanghai Jiao Tong University. We thank Yinfang Lv team (Shanghai Xinying Biotechnology Co. LTD) for animal sample collection. We thank instrumental analysis center of Shanghai Jiao Tong University for assistance with the SCFAs determination.

Conflicts of Interest: The authors declare no conflict of interest.

\section{References}

1. Yanovski, S.Z. and Yanovski, J.A. Long-term Drug Treatment for Obesity: A Systematic and Clinical Review. JAMA. 2014, 311, 74-86.

2. Newman, C.B.; Preiss, D.; Tobert, J.A.; Jacobson, T.A.; Page II, R.L.; Goldstein, L.B.; Chin, C.; Tannock, L.R.; Miller, M.; Raghuveer, G.; et al. Statin Safety and Associated Adverse Events: A Scientific Statement From the American Heart Association. Arteriosclerosis Thrombosis and Vascular Biology. 2018, 38-81. 
3. Bray, G.A.; Frühbeck, G.; Ryan, D.H.; Wilding, J.P.H. Management of obesity. The Lancet. 2016, 387, 19471956.

4. Martel, J.; Ojcius, D.M.; Chang, C.-J.; Lin, C.-S.; Lu, C.-C.; Ko, Y.-F.; Tseng, S.-F.; Lai, H.-C.; Young, J.D. Antiobesogenic and antidiabetic effects of plants and mushrooms. Nature Reviews Endocrinology. 2017, 13, 149-160. 5. Yang, X.; Mo, W.; Zheng, C.; Li, W.; Tang, J.; Wu, X. Alleviating effects of noni fruit polysaccharide on hepatic oxidative stress and inflammation in rats under a high-fat diet and its possible mechanisms. Food \& Function. 2020, 11, 2953-2968.

6. Zhu, K.; Mao, G.; Wu, D.; Yu, C.; Cheng, H.; Xiao, H.; Ye, X.; Linhardt, R.J.; Orfila, C.; Chen, S. Highly Branched RG-I Domain Enrichment Is Indispensable for Pectin Mitigating against High-Fat Diet-Induced Obesity. Journal of Agricultural and Food Chemistry. 2020, 68, 8688-8701.

7. Chen, J.; Liu, J.; Yan, C.; Zhang, C.; Pan, W.; Zhang, W.; Lu, Y.; Chen, L.; Chen, Y. Sarcodon aspratus polysaccharides ameliorated obesity-induced metabolic disorders and modulated gut microbiota dysbiosis in mice fed a high-fat diet. Food \& Function. 2020, 11, 2588-2602.

8. $\quad$ Chang, C.-J.; Lin, C.-S.; Lu, C.-C.; Martel, J.; Ko, Y.-F.; Ojcius, D.M.; Tseng, S.-F.; Wu, T.-R.; Chen, Y.-Y.M.; Young, J.D.; et al. Ganoderma lucidum reduces obesity in mice by modulating the composition of the gut microbiota. Nature Communications. 2017, 8, 16130.

9. Wu, T.R.; Lin, C.S.; Chang, C.J.; Lin, T.L.; Martel, J.; Ko, Y.F.; Ojcius, D.M.; Lu, C.C.; Young, J.D.; Lai, H.C. Gut commensal Parabacteroides goldsteinii plays a predominant role in the anti-obesity effects of polysaccharides isolated from Hirsutella sinensis. Gut. 2018, gutjnl-2017-315458.

10. Huang, Z.-R.; Deng, J.-C.; Li, Q.-Y.; Cao, Y.-J.; Lin, Y.-C.; Bai, W.-D.; Liu, B.; Rao, P.-F.; Ni, L.; Lv, X.-C. Protective Mechanism of Common Buckwheat (Fagopyrum esculentum Moench.) against Nonalcoholic Fatty Liver Disease Associated with Dyslipidemia in Mice Fed a High-Fat and High-Cholesterol Diet. Journal of Agricultural and Food Chemistry. 2020, 68, 6530-6543.

11. Xiong, Q.; Zhu, L.; Zhang, F.; Li, H.; Wu, J.; Liang, J.; Yuan, J.; Shi, Y.; Zhang, Q.; Hu, Y. Protective activities of polysaccharides from Cipangopaludina chinensis against high-fat-diet-induced atherosclerosis via regulating gut microbiota in ApoE-deficient mice. Food \& Function. 2019, 10, 6644-6654.

12. Hopping, K.A.; Chignell, S.M.; Lambin, E.F. The demise of caterpillar fungus in the Himalayan region due to climate change and overharvesting. Proceedings of the National Academy of Sciences. 2018, 115, 11489.

13. Yu, R.; Ye, B.; Yan, C.; Song, L.; Zhang, Z.; Yang, W.; Zhao, Y. Fingerprint analysis of fruiting bodies of cultured Cordyceps militaris by high-performance liquid chromatography-photodiode array detection. Journal of Pharmaceutical \& Biomedical Analysis. 2007, 44, 818-823.

14. Jing, Y.; Cui, X.; Chen, Z.; Huang, L.; Song, L.; Liu, T.; Lv, W.; Yu, R. Elucidation and biological activities of a new polysaccharide from cultured Cordyceps militaris. Carbohydrate polymers. 2014, 102, 288-296.

15. Liao, Y.; Ling, J.; Zhang, G.; Liu, F.; Tao, S.; Han, Z.; Chen, S.; Chen, Z.; Le, H. Cordycepin induces cell cycle arrest and apoptosis by inducing DNA damage and up-regulation of p53 in Leukemia cells. Cell Cycle. 2015, 14, 761-771.

16. Chen, D.-D.; Xu, R.; Zhou, J.-Y.; Chen, J.-Q.; Wang, L.; Liu, X.-S.; Liang, C.-1.; Liu, B.-H.; Lu, R.-R.; Wu, J.-B.; et al. Cordyceps militaris polysaccharides exerted protective effects on diabetic nephropathy in mice via regulation of autophagy. Food \& Function. 2019, 10, 5102-5114.

17. Liu, J.-y.; Feng, C.-p.; Li, X.; Chang, M.-c.; Meng, J.-l.; Xu, L.-j. Immunomodulatory and antioxidative activity of Cordyceps militaris polysaccharides in mice. International Journal of Biological Macromolecules. 2016, 86, 594-598.

18. An, Y.; Li, Y.; Wang, X.; Chen, Z.; Xu, H.; Wu, L.; Li, S.; Wang, C.; Luan, W.; Wang, X.; et al. Cordycepin reduces weight through regulating gut microbiota in high-fat diet-induced obese rats. Lipids in Health and Disease. 2018, 17, 276.

19. Qi, G.; Zhou, Y.; Zhang, X.; Yu, J.; Li, X.; Cao, X.; Wu, C.; Guo, P. Cordycepin promotes browning of white adipose tissue through an AMP-activated protein kinase (AMPK)-dependent pathway. Acta Pharmaceutica Sinica B. 2019, 9, 135-143.

20. Kim, S.B.; Ahn, B.; Kim, M.; Ji, H.-J.; Shin, S.-K.; Hong, I.P.; Kim, C.Y.; Hwang, B.Y.; Lee, M.K. Effect of Cordyceps militaris extract and active constituents on metabolic parameters of obesity induced by high-fat diet in C58BL/6J mice. Journal of Ethnopharmacology. 2014, 151, 478-484.

21. Le Chatelier, E.; Nielsen, T.; Qin, J.; Prifti, E.; Hildebrand, F.; Falony, G.; Almeida, M.; Arumugam, M.; Batto, J.-M.; Kennedy, S.; et al. Richness of human gut microbiome correlates with metabolic markers. Nature. 2013, 500, 541-546. 
22. Chen, L.; Zhang, L.; Wang, W.; Qiu, W.; Liu, L.; Ning, A.; Cao, J.; Huang, M.; Zhong, M. Polysaccharides isolated from Cordyceps Sinensis contribute to the progression of NASH by modifying the gut microbiota in mice fed a high-fat diet. PLOS ONE. 2020, 15, e0232972.

23. Jiaojiao, Z.; Fen, W.; Kuanbo, L.; Qing, L.; Ying, Y.; Caihong, D. Heat and light stresses affect metabolite production in the fruit body of the medicinal mushroom Cordyceps militaris. Applied Microbiology and Biotechnology. 2018, 102, 4523-4533.

24. Liu, X.-C.; Li, H.; Kang, T.; Zhu, Z.-Y.; Pan, L.-c. The effect of fermentation conditions on the structure and anti-tumor activity of polysaccharides from Cordyceps gunnii. RSC Advances. 2019, 9, 18205-18216.

25. Wang, C.-C.; Wu, J.-Y.; Chang, C.-Y.; Yu, S.-T.; Liu, Y.-C. Enhanced exopolysaccharide production by Cordyceps militaris using repeated batch cultivation. Journal of Bioscience and Bioengineering. 2019, 127, 499505.

26. Rayman, M.P. Selenium and human health. The Lancet. 2012, 379, 1256-1268.

27. Zhao, D.; Gao, F.; Zhu, H.; Qian, Z.; Mao, W.; Yin, Y.; Chen, D. Selenium-enriched Bifidobacterium longum DD98 relieves metabolic alterations and liver injuries associated with obesity in high-fat diet-fed mice. Journal of Functional Foods. 2020, 72, 104051.

28. Surhio, M.M.; Wang, Y.; Xu, P.; Shah, F.; Li, J.; Ye, M. Antihyperlipidemic and hepatoprotective properties of selenium modified polysaccharide from Lachnum sp. International Journal of Biological Macromolecules. 2017, 99, 88-95.

29. Gao, Y.; Xu, Y.; Ruan, J.; Yin, J. Selenium affects the activity of black tea in preventing metabolic syndrome in high-fat diet-fed Sprague-Dawley rats. Journal of the ence of Food and Agriculture. 2020, 100, 225-234.

30. Michel. DuBois, K.A.G., J.K. Hamilton, P.A. Rebers, and Fred. Smith. Colorimetric method for determination of sugars and related substances. Analytical Chemistry. 1956, 28, 350-356.

31. J., K. New method for the determination of nitrogen. Chem News. 1883, 48, 101-102.

32. Luque-García, J.L. and Luque de Castro, M.D. Ultrasound-assisted Soxhlet extraction: an expeditive approach for solid sample treatment: Application to the extraction of total fat from oleaginous seeds. Journal of Chromatography A. 2004, 1034, 237-242.

33. Gilani, G.S. and Peace, R.W.J.J.o.A.I. Chromatographic determination of amino acids in foods. $2005,88$.

34. Li, S.; Li, J.; Zhu, Z.; Cheng, S.; He, J.; Lamikanra, O. Soluble dietary fiber and polyphenol complex in lotus root: Preparation, interaction and identification. Food Chemistry. 2020, 314, 126219.

35. Folin, O. and Ciocalteu, V.J.J.o.B.C. ON TYROSINE AND TRYPTOPHANE DETERMINATIONS IN PROTEINS. 1927, 73, 627-650.

36. Pradeep, C.R. and Kuttan, G. Piperine is a potent inhibitor of nuclear factor-kB (NF-kB), c-Fos, CREB, ATF2 and proinflammatory cytokine gene expression in B16F-10 melanoma cells. Internat Immunopharmacol. 2004, 4.

37. Kraus, D.; Yang, Q.; Kahn, B.B. Lipid Extraction from Mouse Feces. Bio-protocol. 2015, 5, e1375.

38. Simpson, E.H. Measurement of Diversity. Nature. 1949, 163, 688-688.

39. Pielou, E.C. The measurement of diversity in different types of biological collections. Journal of Theoretical Biology. 1966, 13, 131-144.

40. Ramette, A. Multivariate analyses in microbial ecology. 2007, 62, 142-160.

41. Anderson, M.J. and Walsh, D.C.I.J.E.M. PERMANOVA, ANOSIM, and the Mantel test in the face of heterogeneous dispersions: What null hypothesis are you testing? 2013.

42. Bokulich, N.A.; Kaehler, B.D.; Rideout, J.R.; Dillon, M.; Bolyen, E.; Knight, R.; Huttley, G.A.; Gregory Caporaso, J. Optimizing taxonomic classification of marker-gene amplicon sequences with QIIME 2's q2-featureclassifier plugin. Microbiome. 2018, 6, 90.

43. DeSantis, T.Z.; Hugenholtz, P.; Larsen, N.; Rojas, M.; Brodie, E.L.; Keller, K.; Huber, T.; Dalevi, D.; Hu, P.; Andersen, G.L. Greengenes, a Chimera-Checked 16S rRNA Gene Database and Workbench Compatible with ARB. Applied and Environmental Microbiology. 2006, 72, 5069-5072.

44. Segata, N.; Izard, J.; Waldron, L.; Gevers, D.; Miropolsky, L.; Garrett, W.S.; Huttenhower, C. Metagenomic biomarker discovery and explanation. Genome Biology. 2011, 12, R60.

45. Zhang, Y.; Zeng, Y.; Cui, Y.; Liu, H.; Dong, C.; Sun, Y. Structural characterization, antioxidant and immunomodulatory activities of a neutral polysaccharide from Cordyceps militaris cultivated on hull-less barley. Carbohydrate Polymers. 2020, 235, 115969.

46. Kajimura, S.; Spiegelman, Bruce M.; Seale, P. Brown and Beige Fat: Physiological Roles beyond Heat Generation. Cell Metabolism. 2015, 22, 546-559. 
47. Koh, A.; De Vadder, F.; Kovatcheva-Datchary, P.; Bäckhed, F. From Dietary Fiber to Host Physiology: ShortChain Fatty Acids as Key Bacterial Metabolites. Cell. 2016, 165, 1332-1345.

48. Sanna, S.; van Zuydam, N.R.; Mahajan, A.; Kurilshikov, A.; Vich Vila, A.; Võsa, U.; Mujagic, Z.; Masclee, A.A.M.; Jonkers, D.M.A.E.; Oosting, M.; et al. Causal relationships among the gut microbiome, short-chain fatty acids and metabolic diseases. Nature Genetics. 2019, 51, 600-605.

49. Li, X.; Wang, H.; Wang, T.; Zheng, F.; Wang, H.; Wang, C. Dietary wood pulp-derived sterols modulation of cholesterol metabolism and gut microbiota in high-fat-diet-fed hamsters. Food \& Function. 2019, 10, 775-785.

50. Nguyen; Khoi; Song; Tran; Goon-Tae; Kim; Si-Hyun; Park; Dongyup; Lee. Fermented Cordyceps militaris Extract Prevents Hepatosteatosis and Adipocyte Hypertrophy in High Fat Diet-Fed Mice. Nutrients. 2019.

51. Ren, D.; Wang, N.; Guo, J.; Yuan, L.; Yang, X. Chemical characterization of Pleurotus eryngii polysaccharide and its tumor-inhibitory effects against human hepatoblastoma HepG-2 cells. Carbohydrate Polymers. 2016, 138, 123-133.

52. Rayman, M.P. Selenium and human health. Lancet. 2012, 379.

53. Luo, J.; Yang, H.; Song, B.-L. Mechanisms and regulation of cholesterol homeostasis. Nature Reviews Molecular Cell Biology. 2020, 21, 225-245.

54. Sandesara, P.B.; Virani, S.S.; Fazio, S.; Shapiro, M.D. The Forgotten Lipids: Triglycerides, Remnant Cholesterol, and Atherosclerotic Cardiovascular Disease Risk. Endocrine Reviews. 2018, 40, 537-557.

55. Barter, P. HDL-C: Role as a risk modifier. Atherosclerosis Supplements. 2011, 12, 267-270.

56. Wu, Q.; Wang, Q.; Fu, J.; Ren, R. Polysaccharides derived from natural sources regulate triglyceride and cholesterol metabolism: a review of the mechanisms. Food \& Function. 2019, 10, 2330-2339.

57. Stefan, N.; Häring, H.-U.; Schulze, M.B. Metabolically healthy obesity: the low-hanging fruit in obesity treatment? The lancet. Diabetes \& endocrinology. 2018, 6, 249-258.

58. Ahima, R.S. and Lazar, M.A. The Health Risk of Obesity-Better Metrics Imperative. Science. 2013, 341, 856-858.

59. Bäckhed, F.; Ding, H.; Wang, T.; Hooper, L.V.; Koh, G.Y.; Nagy, A.; Semenkovich, C.F.; Gordon, J.I. The gut microbiota as an environmental factor that regulates fat storage. Proceedings of the National Academy of Sciences of the United States of America. 2004, 101, 15718-15723.

60. Li, S.; Li, J.; Mao, G.; Wu, T.; Hu, Y.; Ye, X.; Tian, D.; Linhardt, R.J.; Chen, S. A fucoidan from sea cucumber Pearsonothuria graeffei with well-repeated structure alleviates gut microbiota dysbiosis and metabolic syndromes in HFD-fed mice. Food \& Function. 2018, 9, 5371-5380.

61. Lu, Y.; Zhao, A.; Wu, Y.; Zhao, Y.; Yang, X. Soybean soluble polysaccharides enhance bioavailability of genistein and its prevention against obesity and metabolic syndrome of mice with chronic high fat consumption. Food \& Function. 2019, 10, 4153-4165.

62. Kasaikina, M.V.; Kravtsova, M.A.; Lee, B.C.; Seravalli, J.; Peterson, D.A.; Walter, J.; Legge, R.; Benson, A.K.; Hatfield, D.L.; Gladyshev, V.N. Dietary selenium affects host selenoproteome expression by influencing the gut microbiota. 2011, 25, 2492-2499.

63. Jing, N.; Liu, X.; Jin, M.; Yang, X.; Hu, X.; Li, C.; Zhao, K. Fubrick tea attenuates high-fat diet induced fat deposition and metabolic disorder by regulating gut microbiota and caffeine metabolism. Food \& Function. 2020, 11, 6971-6986.

64. Anhê, F.F.; Nachbar, R.T.; Varin, T.V.; Trottier, J.; Dudonné, S.; Le Barz, M.; Feutry, P.; Pilon, G.; Barbier, O.; Desjardins, Y.; et al. Treatment with camu camu (<em $>$ Myrciaria dubia $</$ em $>$ ) prevents obesity by altering the gut microbiota and increasing energy expenditure in diet-induced obese mice. Gut. 2019, 68, 453.

65. Li, F.; Jiang, C.; Krausz, K.W.; Li, Y.; Albert, I.; Hao, H.; Fabre, K.M.; Mitchell, J.B.; Patterson, A.D.; Gonzalez, F.J. Microbiome remodelling leads to inhibition of intestinal farnesoid $\mathrm{X}$ receptor signalling and decreased obesity. Nature Communications. 2013, 4, 2384.

66. Tang, D.; Wang, Y.; Kang, W.; Zhou, J.; Dong, R.; Feng, Q. Chitosan attenuates obesity by modifying the intestinal microbiota and increasing serum leptin levels in mice. Journal of Functional Foods. 2020, 64, 103659.

67. Han, Y.; Song, M.; Gu, M.; Ren, D.; Zhu, X.; Cao, X.; Li, F.; Wang, W.; Cai, X.; Yuan, B.; et al. Dietary Intake of Whole Strawberry Inhibited Colonic Inflammation in Dextran-Sulfate-Sodium-Treated Mice via Restoring Immune Homeostasis and Alleviating Gut Microbiota Dysbiosis. Journal of Agricultural and Food Chemistry. 2019, 67, 9168-9177.

68. Raza, G.S.; Maukonen, J.; Makinen, M.; Niemi, P.; Niiranen, L.; Hibberd, A.A.; Poutanen, K.; Buchert, J.; Herzig, K.-H. Hypocholesterolemic Effect of the Lignin-Rich Insoluble Residue of Brewer's Spent Grain in Mice Fed a High-Fat Diet. Journal of Agricultural and Food Chemistry. 2019, 67, 1104-1114. 
69. Zeng, H.; Ishaq, S.L.; Zhao, F.-Q.; Wright, A.-D.G. Colonic inflammation accompanies an increase of $\beta$ catenin signaling and Lachnospiraceae/Streptococcaceae bacteria in the hind gut of high-fat diet-fed mice. The Journal of Nutritional Biochemistry. 2016, 35, 30-36. 